\title{
Increased susceptibility to hypertensive renal disease in streptozotocin-treated diabetic rats is not modulated by salt intake
}

\author{
C. A. Sima • M. P. Koeners • J. A. Joles • B. Braam • \\ A. B. Magil $\cdot$ W. A. Cupples
}

Received: 2 January 2012 / Accepted: 2 April 2012 /Published online: 6 May 2012

(C) Springer-Verlag 2012

\begin{abstract}
Aims/hypothesis In early type 1 diabetes mellitus, renal salt handling is dysregulated, so that the glomerular filtration rate becomes inversely proportional to salt intake. The salt paradox occurs in both humans and rats and, with low salt intake, results in diabetic hyperfiltration. We tested whether increased salt intake could reduce the susceptibility to injury of non-clipped kidneys in diabetic rats with pre-existing Goldblatt hypertension.

Methods Male Long-Evans rats were made hypertensive and half were then made diabetic. Blood glucose was maintained at $\sim 20-25 \mathrm{mmol} / \mathrm{l}$ by insulin implants. One half of each received only the salt in normal chow ( $1 \%$ by weight) and the other half received added salt in drinking water to
\end{abstract}

C. A. Sima $\cdot$ M. P. Koeners $\cdot$ W. A. Cupples

Department of Biology, University of Victoria,

Victoria, BC, Canada

M. P. Koeners · J. A. Joles

Department of Nephrology \& Hypertension,

University Medical Center,

Utrecht, the Netherlands

B. Braam

Departments of Medicine \& Physiology, University of Alberta,

Edmonton, AB, Canada

A. B. Magil

Department of Pathology \& Laboratory Medicine,

St Paul's Hospital, University of British Columbia,

Vancouver, BC, Canada

W. A. Cupples $(\triangle)$

Department of Biomedical Physiology \& Kinesiology,

Simon Fraser University,

8888 University Drive,

Burnaby, BC, Canada V5A 1S6

e-mail: wcupples@sfu.ca equal $2.7 \%$ by weight of food intake. Weekly $24 \mathrm{~h}$ blood pressure records were acquired by telemetry during the 4month experiment.

Results Systolic blood pressure was not affected by diabetes or increased salt intake, alone or together. Autoregulation was highly efficient in the non-clipped kidney of both intact and diabetic rats. Histological examination showed minor injury in the clipped kidney, which did not differ among groups. The non-clipped kidney showed extensive pressuredependent glomerular and vascular injury in both intact and diabetic rats.

Conclusions/interpretation The relationship between pressure and injury was shifted toward lower blood pressure in diabetic rats, indicating that diabetes increased the susceptibility of the kidney to injury despite preservation of autoregulation. The increased susceptibility was not affected by high salt intake in the diabetic rats, thus disproving the hypothesis.

Keywords Autoregulation · Hypertension - Kidney ·

Nephropathy $\cdot$ Rats $\cdot$ Renal blood flow $\cdot$ Susceptibility

Type 1 diabetes mellitus

$\begin{array}{ll}\text { Abbreviations } & \\ \text { 2K1C } & \text { 2 Kidney 1 clip (Goldblatt) hypertension } \\ \text { ANCOVA } & \text { Analysis of covariance } \\ \text { ANG II } & \text { Angiotensin II } \\ \text { BP } & \text { Blood pressure } \\ \text { DM-H } & \text { Diabetic-high salt } \\ \text { DM-N } & \text { Diabetic-normal salt } \\ \text { IN-H } & \text { Intact-high salt } \\ \text { IN-N } & \text { Intact-normal salt } \\ \text { NOS } & \text { Nitric oxide synthase } \\ \text { RBF } & \text { Renal blood flow } \\ \text { STZ } & \text { Streptozotocin } \\ \text { TG feedback } & \text { Tubuloglomerular feedback }\end{array}$




\section{Introduction}

Diabetes mellitus and hypertension account for the great majority of chronic kidney disease [1]. Hypertension is generally accepted to be a major contributor to the development of nephropathy in diabetes [2, 3] and accelerates all other forms of nephropathy [4]. There is also considerable evidence for the importance of genetic and functional factors in determining target organ susceptibility [5-7]. In uncomplicated type 1 or type 2 diabetes, there is a high incidence of diabetic hyperfiltration, which results from glomerular hypertension, presumably because of inappropriately low pre-glomerular resistance [2, 8]. Only one mechanism, autoregulation, protects glomeruli from hypertension and blood pressure (BP) fluctuations [9]. Reduced efficiency of autoregulation increases susceptibility to, and rate of progression of, hypertensive nephropathy [9-11]. It is often considered that autoregulation is impaired in diabetes, and diabetic hyperfiltration has been attributed to enhanced transmission of systemic pressure to glomeruli [2, 12, 13]. However, efficient autoregulation has also been reported in diabetes [7, 14-16], and the literature has been described as contradictory [14, 17].

GFR is almost always independent of, or varies directly with, salt intake. However, both rats and humans with type 1 diabetes exhibit an inverse relationship between dietary salt and GFR, a phenomenon known as the salt paradox [18-20]. Vallon and colleagues have investigated the salt paradox, and from their results derived the tubulocentric hypothesis [12]. This holds that the problem originates in a dysregulated increase in fractional proximal tubular reabsorption, so that salt delivery to the macula densa is reduced. Tubuloglomerular feedback (TG feedback) then causes afferent vasodilatation and glomerular hyperfiltration. Increasing salt intake reduces fractional proximal salt reabsorption followed by TG feedback-mediated afferent constriction and reduction of GFR and, importantly, of glomerular capillary pressure $[12,21]$. The existence of the salt paradox suggests the possibility of adjusting salt intake to increase preglomerular resistance, thereby reducing nephropathy in diabetes [21], a position that is not consistent with the widespread belief that a low-sodium diet is universally beneficial.

To determine consequences of increased salt intake on susceptibility to (hypertensive) renal injury, it is first necessary to consider other consequences of altered salt intake. More formally, one must test whether a manipulation affects the input variable(s) and whether it affects target organ susceptibility. Salt-sensitive hypertension is well known in some humans and other animals [22], and a high incidence of salt sensitivity of BP has been suggested in the evolution of diabetes mellitus [23], although this too is not a universal finding [24]. Therefore, we first showed that increasing salt intake from $1 \%$ to $3 \%$ by weight of food eaten did not affect $\mathrm{BP}$ in intact rats or in poorly controlled type 1 diabetes [16]. The salt paradox was confirmed, but since no pathological changes were seen, that study did not test whether increasing salt intake affected susceptibility to nephropathy in type 1 diabetes.

The specific predictions tested in this study were that type 1 diabetes would increase susceptibility to hypertensive renal injury and that moderately increased salt intake would restore normal susceptibility. The subsidiary hypothesis was that the effects of type 1 diabetes and type 1 diabetes plus salt on renal susceptibility would occur through changes in the efficiency of autoregulation.

\section{Methods}

The hypertension model (2 kidney, 1 clip [2K1C]) and the strain of rat were chosen for specific reasons. In the $2 \mathrm{~K} 1 \mathrm{C}$ model, the two kidneys are exposed to different pressure burdens, but identical glycaemia, which provides a qualitative control for hypertensive renal injury. It also generates a wide range of $\mathrm{BP}$, which is a desired feature of the model for this study. Pressure dependency of renal disease is assessed by plotting incidence of injury against BP and then testing whether the best fit lines relating injury to pressure differ significantly [25]. Consequently, the aim was to achieve similar average systolic pressures and the widest possible range of pressure in each group. The Long-Evans rat was chosen because it is the parent strain of the Otsuka Long-Evans Tokushima fatty rat. Males of that strain reliably develop type 2 diabetes and exhibit classic diabetic nephropathy including nodular lesions of glomeruli plus coincident hypertension [26]. Thus, there was a reasonable expectation that the parental strain would display diabetic nephropathy within the experimental duration, but also recognition that progression of hypertensive disease is much more rapid than progression of diabetic nephropathy.

All procedures involving animals were approved by the University of Victoria Animal Care Committee and were consistent with the Guidelines promulgated by the Canadian Council on Animal Care. Male rats were acquired from Charles River (Canada) (St Constant, QC, Canada) and housed individually. They were allowed free access to drinking fluid and to Purina LabDiet 5001 containing sodium and chloride $0.4 \%$ and $0.57 \%$ by weight, respectively. Procedures were initiated when the rats were 10 weeks old. All surgical procedures were performed under isoflurane anaesthesia with analgesia (buprenorphine, Temgesic; Reckitt \& Benckiser, Mississauga, ON, Canada; $0.01 \mathrm{mg} / \mathrm{kg}$, i.p. twice daily for 2 days) and, except in the terminal study, under sterile conditions. 
A PA11-C40 pressure telemeter (Data Sciences International, St Paul, MN, USA) was implanted s.c. in the left flank and secured by a purse string suture. The $10 \mathrm{~cm}$ cannula was inserted in the femoral artery and advanced into the aorta to the level of the renal arteries, leaving slack to accommodate growth $[16,27]$. After recovery ( 2 weeks) and acquisition of a control record, all rats were subjected to the $2 \mathrm{~K} 1 \mathrm{C}$ procedure [27]. After recovery ( 2 weeks) and acquisition of another data record, rats were assigned to one of four experimental groups: intact with normal salt (IN-N), intact with high salt (IN-H), diabetic with normal salt (DM-N), diabetic with high salt (DM-H). Then type 1 diabetes was induced by injection of $60 \mathrm{mg} / \mathrm{kg}$ streptozotocin (STZ; Pfizer Canada, Kirkland, QC, Canada) in ice-cold saline $(154 \mathrm{mmol} / \mathrm{l} \mathrm{NaCl})$ i.p. (1st cohort) or i.v. (2nd cohort) under light anaesthesia. Three weeks later, rats were assigned to normal (1\%) or high salt (2.7\%) intake. A total of 38 rats entered the protocol including eight IN-N, eight $\mathrm{IN}-\mathrm{H}$, ten DM-N and $12 \mathrm{DM}-\mathrm{H}$. Five rats (one IN-N, two DM-N, two DM-H) were censored before the eighth week of the high-salt period and excluded from analysis. The experimental design is presented in Fig. 1.

Chronic data acquisition Metabolic measurements $(24 \mathrm{~h}$ food and fluid intake, urine output) plus $24 \mathrm{~h}$ BP records were acquired at control, after $2 \mathrm{~K} 1 \mathrm{C}$, and weekly thereafter. Data were acquired at $1,000 \mathrm{~Hz}$ in $10 \mathrm{~s}$ bursts every $2 \mathrm{~min}$. Systolic BP is reported in order to maintain consistency with

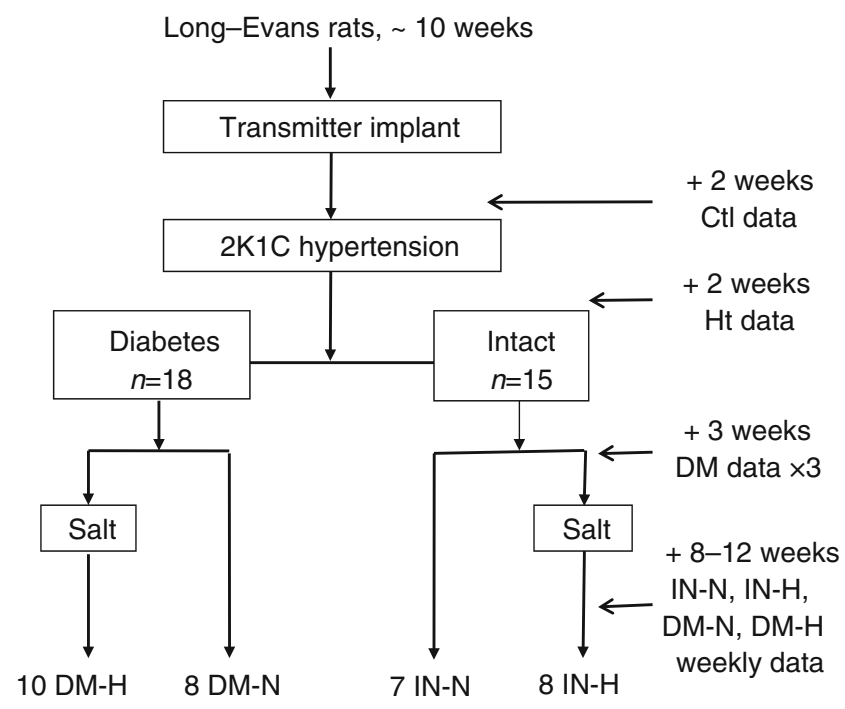

Fig. 1 Summary of experimental design. Groups include intact rats with normal salt intake (IN-N), intact rats with high salt intake (IN-H), diabetic rats with normal salt intake (DM-N), and diabetic rats with high salt intake (DM-H). Control (Ctl) data (24 h BP telemetry, $24 \mathrm{~h}$ food intake and urine collection) were acquired 2 weeks after implanting transmitters. Hypertensive $(\mathrm{Ht})$ data were acquired 2 weeks after the $2 \mathrm{~K} 1 \mathrm{C}$ procedure. Diabetic (DM) data were acquired for 3 weeks after STZ treatment previous studies in the literature $[7,14,16]$ and because it is more closely correlated with organ damage than mean or diastolic BP [10]. Body weight and blood glucose were measured twice weekly in all animals; blood samples were acquired from the tail of conscious, unrestrained rats. When blood glucose exceeded $30 \mathrm{mmol} / 1$ (Ascensia glucometer ACCU-Check; Boehringer, Burlington, ON, Canada), one half of a LinPlant (Linshin Canada, Toronto, ON, Canada) was implanted s.c. LinPlants were renewed as needed at $\sim 4-$ week intervals. Urinary protein (Bradford assay), $\mathrm{Na}^{+}$and $\mathrm{K}^{+}$concentrations were determined at the Department of Nephrology \& Hypertension of the University Medical Center Utrecht, the Netherlands [28]. Body composition was estimated from body length (snout to anus; centimetre) and the ratio of weight to length at the end of the experiment.

Autoregulation At the end of the study, renal blood flow (RBF) and autoregulation were assessed in the left, nonclipped kidney [16, 29]. Briefly, after anaesthesia and cannulation of the trachea, cannulae were placed in a femoral artery to measure perfusion pressure and in a femoral vein for infusions. Arterial pressure was measured by a pressure transducer driven by a Kent (Torrington, CT, USA) TRN050 amplifier, and RBF was measured by a Transonic Systems (Ithaca, NY, USA) T401 transit time ultrasound flow meter (1PRB probe). The rat received a constant i.v. infusion of $2 \%$ charcoal-washed BSA (Sigma-Aldrich, Oakville, ON, Canada) in Ringer solution at $0.6 \%$ of body weight per hour. In diabetic rats the infusion was adjusted to match the urine flow. A servo-controlled occluder was placed on the aorta between the right and left renal arteries and was used to adjust renal perfusion pressure. After $60 \mathrm{~min}$ equilibration, baseline RBF and perfusion pressure were acquired, then renal perfusion pressure was reduced in steps of $10 \mathrm{mmHg} \times 1 \mathrm{~min}$ to $50 \mathrm{mmHg}$ to determine the autoregulatory range [16].

Histological examination At the end of the experiment, both kidneys were harvested, weighed, split, blotted and fixed by immersion in $10 \%$ neutral buffered formalin. After being embedded in paraffin, $4 \mu \mathrm{m}$ sections were stained with periodic acid-Schiff's reagent and counterstained with haematoxylin to show nuclei. The slides were examined by a renal pathologist (A.B.M.) in single blind fashion. A cross-section of each kidney was examined to detect the presence of diabetic and hypertensive microvascular, glomerular and tubulointerstitial lesions. The glomerular changes were expressed as the percentage of glomeruli showing injury. Estimation of tubular atrophy and interstitial fibrosis used a $0-3$ scale $(0=0-5 \%$ of cortex displaying injury, $1=5-25 \%$ injured, $2=25-50 \%$ injured, $3 \geq 50 \%$ injured) to assess chronic parenchymal pathology. Mesangial expansion was modest and did not differ among groups. 
Statistical analysis One-way and two-way ANOVA, with or without repeated measures, was used to analyse the interaction of time and treatment among the groups. ANOVAs were completed by planned contrasts. Post hoc completion used Tukey's HSD test. Although systolic pressure in the diabetic rats was not significantly different from that in the intact rats, the average values were numerically lower in diabetic than in intact groups. To allow for this potential confound, systolic pressure was used as a covariate in analysis of covariance (ANCOVA) exploring progression of proteinuria. Linear regression was used to analyse the relationship between glomerulosclerosis and BP. Data are presented as means $\pm \mathrm{SEM}$, and $p<0.05$ is considered to indicate a significant difference.

\section{Results}

Body weight, presented in Fig. 2a, increased progressively in both intact groups. Body weight in the two diabetic groups diverged from the intact groups after STZ $(p<0.01)$ and increased very slowly thereafter. As shown in Fig. 2b, blood glucose in intact rats was $4.5 \pm 0.1 \mathrm{mmol} / \mathrm{l}$, whereas in diabetic rats it was maintained at $23 \pm 1 \mathrm{mmol} / \mathrm{l}(p<0.01)$ by insulin implants. Salt intake had no effect on body weight or blood glucose in intact or diabetic rats. Table 1 shows that final body length was slightly less $(p<0.01)$ in diabetic than intact rats, while weight/length ratio was substantially less in diabetic rats $(p<0.01)$, indicating that body composition differed substantially, precluding normalisation of the data to body weight [30]. Neither variable was affected by salt intake. The duration of the experiment from the $2 \mathrm{~K} 1 \mathrm{C}$ procedure to killing the rats did not differ between intact and diabetic rats $(18.1 \pm 0.5$ versus $17.2 \pm 0.5$ weeks, respectively). The weight of the non-clipped kidney was greater in diabetic than intact rats $(p<0.05)$ and the weight of the nonclipped kidney was greater than that of the clipped kidney in all groups $(p<0.01)$. One atrophic clipped kidney was found in each group, and these were not included in the analysis. Neither clipped nor non-clipped kidney weight varied with salt intake.

All rats were normotensive at the control recording and hypertensive after the $2 \mathrm{~K} 1 \mathrm{C}$ procedure (Fig. 2c). Owing to the wide range of systolic pressure within each group, systolic pressure did not differ among the four groups at any time. Systolic pressure was not affected by salt intake at any time in intact or diabetic rats. A very similar pattern was seen for diastolic pressure (Fig. 2c).

As shown in Fig. 3a, $24 \mathrm{~h} \mathrm{~K}^{+}$excretion was stable in the two intact groups, consistent with stable food consumption. Induction of diabetes increased $\mathrm{K}^{+}$excretion $(p<0.01)$, indicating that the diabetic rats were hyperphagic. Energy intake estimated from $\mathrm{K}^{+}$excretion is consistent with measured
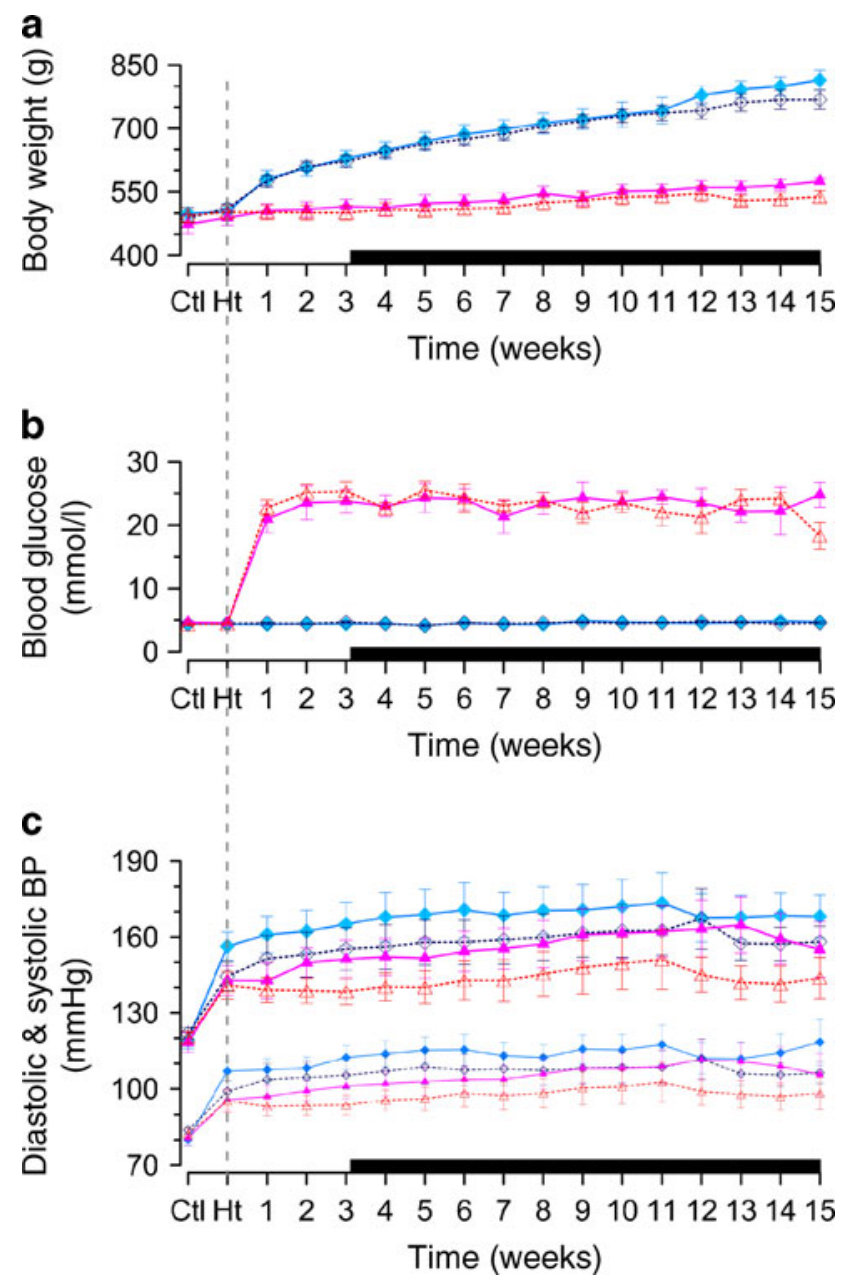

Fig. 2 Body weight (a), blood glucose (b) and diastolic and systolic pressure (c) from the initial $(\mathrm{Ctl})$ and hypertensive $(\mathrm{Ht})$ records through induction of diabetes, denoted by the vertical dashed line, and high salt intake (beginning after week 3 , denoted by the black bar on the abscissa). a Divergent weight gain in intact and diabetic rats is evident. b Stability of blood sugar in intact rats and hyperglycaemia in diabetic rats is apparent. $\mathbf{c}$ Systolic pressures were similar in all groups before and after induction of hypertension, of diabetes, and during high salt intake in $\mathrm{IN}-\mathrm{H}$ and DM-H rats. Diastolic pressures show the same pattern. IN-N $(n=7)$ is denoted by the solid light blue line and solid diamonds, IN-H $(n=8)$ is denoted by the dashed dark blue line and open diamonds, DM-N $(n=8)$ is denoted by the solid magenta line and solid triangles, and DM-H $(n=10)$ is denoted by the dashed red line and open triangles. Data are presented as mean \pm SEM

food intake (not shown). Neither $\mathrm{K}^{+}$excretion nor food intake was affected by salt intake. Figure $3 \mathrm{~b}$ shows that $\mathrm{Na}^{+}$excretion rose modestly due to diabetic hyperphagia $(p<0.01)$. It also displays the greater increase due to salt supplementation.

Figure $3 \mathrm{c}$ illustrates the progressive rise of protein excretion in all groups. Although two-way repeatedmeasures ANOVA did not reveal significant differences among groups, ANCOVA using systolic BP as covariate revealed that protein excretion progressed more rapidly in diabetic rats, being consistently higher than in intact 
Table 1 Body length, body weight/length ratio, kidney weights, anaesthetised $\mathrm{BP}$ and $\mathrm{RBF}$ at the terminal procedure (seven IN-N, eight IN-H, eight DM-N and ten DM-H rats)

\begin{tabular}{lllll}
\hline Terminal data & IN-N & IN-H & DM-N & DM-H \\
\hline Body weight (g) & $798 \pm 45$ & $767 \pm 26$ & $554 \pm 14$ & $543 \pm 19$ \\
Body length (cm) & $28.2 \pm 0.3$ & $28.3 \pm 0.1$ & $27.4 \pm 0.3^{* *}$ & $26.4 \pm 0.4^{* *}$ \\
Weight/length & $28.3 \pm 1.4$ & $27.2 \pm 1.0$ & $20.2 \pm 0.6^{* *}$ & $20.6 \pm 0.5^{* *}$ \\
$\begin{array}{l}\text { Weight of left } \\
\text { kidney (g) }\end{array}$ & $2.90 \pm 0.08$ & $2.98 \pm 0.23$ & $3.47 \pm 0.13^{*}$ & $3.37 \pm 0.26^{*}$ \\
$\begin{array}{l}\text { Weight of right } \\
\text { kidney (g) }\end{array}$ & $2.01 \pm 0.18$ & $2.01 \pm 0.16$ & $2.35 \pm 0.10$ & $2.31 \pm 0.23$ \\
BP (mmHg) & $143 \pm 8$ & $124 \pm 3$ & $138 \pm 11$ & $129 \pm 5$ \\
RBF (ml/min) & $11.1 \pm 2.4$ & $11.4 \pm 1.4$ & $8.7 \pm 0.9$ & $8.0 \pm 1.3$ \\
\hline
\end{tabular}

Data are presented as mean \pm SEM

In each group there was one atrophic right kidney (missing or weight $<0.5 \mathrm{~g}$ ) that is not included in the data presented. In addition, left kidneys were larger than right kidneys, $p<0.05$ in all groups

${ }^{*} p<0.05$ vs IN rats; $* * p<0.01$ vs IN rats

rats $(p<0.05)$ beginning at week 3 , before salt was added to the diet.

RBF and autoregulation were assessed in terminal studies. Mean BP under anaesthesia was not significantly different in the groups; nor did baseline RBF differ among groups (Table 1). Figure 4 shows that autoregulation was efficient in all groups; the slopes of RBF vs renal perfusion pressure were not different from zero or from each other at pressures $>100 \mathrm{mmHg}$. The only significant difference was that $\mathrm{RBF}$ was maintained at lower perfusion pressure in the DM-N group $(p<0.05)$.

Histological results are reported in Table 2. The dominant injury was focal and segmental glomerulosclerosis, with vascular injury in rats with the highest BP. Tubular atrophy and interstitial fibrosis were modest and were not different between intact and diabetic conditions. The clipped kidney showed only minor glomerular lesions, which did not differ between intact and diabetic rats, whereas the non-clipped kidney showed substantial glomerular injury. Figure 5 illustrates glomerular and vascular injuries that exemplify those found in non-clipped kidneys of both intact and diabetic rats. Figure 5a shows segmental glomerulosclerosis in one glomerulus while an adjacent glomerulus appears normal. Figure 5b shows intimal fibrinoid changes typical of accelerated or malignant hypertension.

Pressure dependency of glomerular injury is shown in Fig. 6. ANCOVA with systolic pressure as a covariate showed that the incidence of glomerular injury was greater in diabetic than in intact rats $(p<0.01)$, but that IN-N did not differ from IN-H and nor did DM-N differ from DM-H. The best fit lines to the data were $\%$ glomeruli injured $=(0.44 \pm$ $0.11) \times$ systolic pressure $-(54 \pm 19), r^{2}=0.54$, for intact rats, and $\%$ glomeruli injured $=(0.53 \pm 0.12) \times$ systolic pressure - a
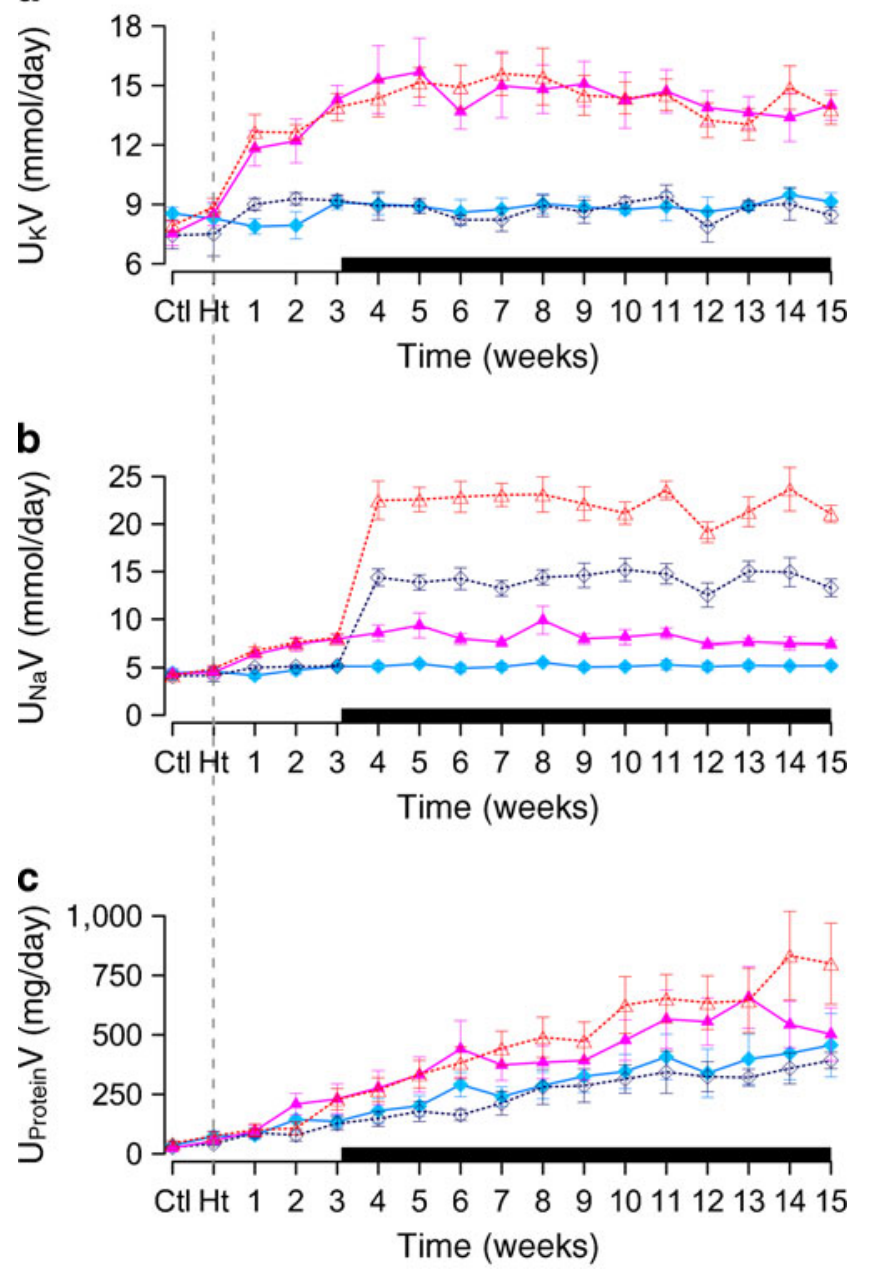

Fig. 3 Urinary potassium excretion $\left(\mathrm{U}_{\mathrm{K}} \mathrm{V}\right)(\mathbf{a})$, urinary sodium excretion $\left(\mathrm{U}_{\mathrm{Na}} \mathrm{V}\right)(\mathbf{b})$ and urinary protein excretion $\left(\mathrm{U}_{\text {Protein }} \mathrm{V}\right)(\mathbf{c})$ from the initial (Ctl) and hypertensive (Ht) records through diabetes (weeks 1, 2, 3), and high salt intake (weeks 4-15). a $\mathrm{U}_{\mathrm{K}} \mathrm{V}$ increased upon induction of type 1 diabetes due to hyperphagia and was stable thereafter. b Diabetic hyperphagia caused a small increase in $U_{\mathrm{Na}} \mathrm{V}$; high salt intake caused a prompt and sustained increase in sodium excretion. $\mathbf{c}$ Protein excretion was similar among groups at $\mathrm{Ctl}$ and $\mathrm{Ht}$ records, increased progressively in all groups, and was greater in diabetic than in intact rats from week 3 onward. Experimental groups and high salt period are identified as in Fig. 2

$(52 \pm 19), r^{2}=0.54$, for diabetic rats. Thus the slope was steeper in diabetic rats. Although the $y$-intercepts did not differ, the $x$-intercepts (the pressure at which there is zero projected injury) differed substantially, being $98 \mathrm{mmHg}$ in the diabetic rats and $123 \mathrm{mmHg}$ in the intact rats.

\section{Discussion}

Diabetic hyperfiltration results largely from inappropriate reduction of pre-glomerular resistance with consequent increase in glomerular capillary pressure, but paradoxically 


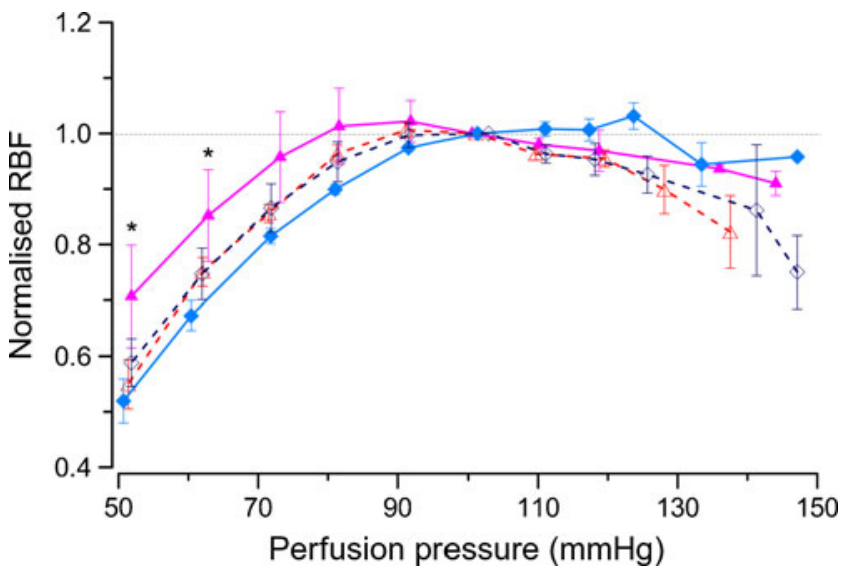

Fig. 4 Steady-state RBF normalised to RBF at $90 \mathrm{mmHg}$ as a function of perfusion pressure. Highly efficient autoregulation was seen in all four groups of rats. The lower limit of autoregulation was extended to lower pressure in DM-N $(p<0.05)$ compared with IN-N and this shift was abrogated by high salt intake in DM-H. Increased salt intake was without effect in intact rats. Experimental groups are identified as in Fig. 2. IN-N $(n=6) ; \mathrm{IN}-\mathrm{H}(n=7) ; \mathrm{DM}-\mathrm{N}(n=6) ; \mathrm{DM}-\mathrm{H}(n=7) .{ }^{*} p<0.05$ DM-N versus IN-N

can be abrogated by increasing salt intake in both humans and rats. Restoration of normal GFR involves reduction of glomerular capillary pressure via increased pre-glomerular resistance. This study tested the hypothesis that increasing salt intake can reduce susceptibility of the diabetic kidney to hypertensive injury. The tubulocentric hypothesis predicts that increasing salt intake will increase pre-glomerular resistance and the autoregulatory capacity of TG feedback. Thus the subsidiary hypothesis was that the diabetic and salt-dependent changes in susceptibility are mediated through changes in autoregulatory efficiency.

Three successive manoeuvres were applied to the animals (2K1C, type 1 diabetes, high salt intake), all known, or presumed, to adversely affect BP and kidneys. A wide and largely overlapping range of systolic pressures was achieved to provide a test for pressure dependency of renal injury. Injection of STZ caused profound and sustained

Table 2 Results of histological examination of non-clipped (N-C) and clipped (C) kidneys

\begin{tabular}{|c|c|c|c|c|c|}
\hline Injury & Clip status & IN-N & IN-H & DM-N & DM-H \\
\hline \multirow{2}{*}{$\begin{array}{l}\text { Glomerular } \\
\text { damage }\end{array}$} & $\mathrm{N}-\mathrm{C}$ & $26 \pm 7$ & $14 \pm 4$ & $30 \pm 7$ & $31 \pm 7$ \\
\hline & $\mathrm{C}$ & $2.8 \pm 1.6$ & $1.7 \pm 0.7$ & $0.9 \pm 0.7$ & $1.3 \pm 0.8$ \\
\hline \multirow{2}{*}{$\begin{array}{l}\text { Tubular atrophy } \\
\text { and interstitial } \\
\text { fibrosis }\end{array}$} & $\mathrm{N}-\mathrm{C}$ & $1.4 \pm 0.2$ & $1.4 \pm 0.2$ & $1.6 \pm 0.2$ & $1.5 \pm 0.2$ \\
\hline & $\mathrm{C}$ & $0.5 \pm 0.5$ & $0.5 \pm 0.3$ & $0.8 \pm 0.5$ & $0.5 \pm 0.5$ \\
\hline
\end{tabular}

Data are presented as mean \pm SEM

Glomerular damage was scored as \% of glomeruli that showed injury. ANOVA showed greater injury in diabetic rats than in intact rats $(p<0.01$, with systolic pressure as covariate). Parenchymal injury (tubular atrophy and interstitial fibrosis) was scored on a scale of $0-3$

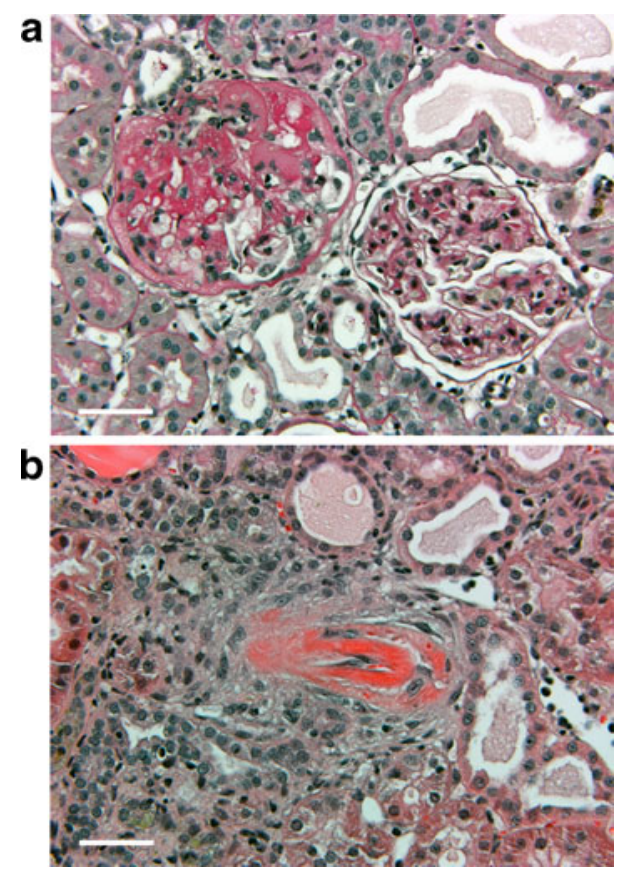

Fig. 5 Common renal pathology. a Segmental glomerulosclerosis in the glomerulus on the left while the glomerulus on the right appears normal. b Intimal fibrinoid changes in an arteriole. In both panels there is little evidence of tubulointerstitial disease. Stained with periodic acid-Schiff's reagent and haematoxylin; scale bars indicate $50 \mu \mathrm{m}$

hyperglycaemia, which required insulin implants to permit growth, albeit at a much reduced rate. Food intake increased with onset of type 1 diabetes and was not affected by increasing salt intake, indicating that there were no

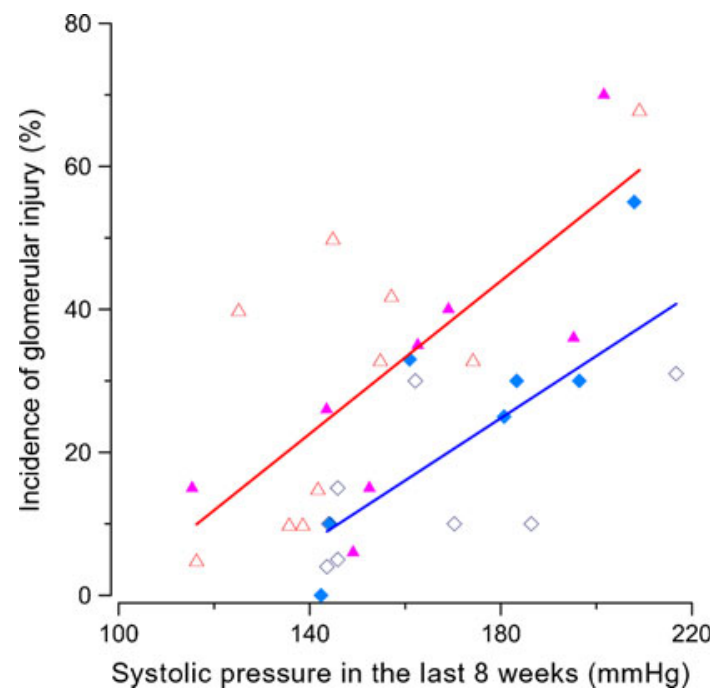

Fig. 6 Pressure dependency of glomerular injury in the non-clipped kidney. Incidence of focal segmental glomerulosclerosis as a function of the pressure load, defined as the average systolic pressure recorded in the last 8 weeks for each rat. Least squares fits to the data for intact $\left(15 ; r^{2}=0.54\right)$ and diabetic $\left(18 ; r^{2}=0.54\right)$ rats show that glomerular injury was worse in diabetic than in intact rats at any pressure $(p<0.01)$. Experimental groups are identified as in Fig. 2 
aversive responses to the added salt load. Collectively, these data show that suitable conditions were achieved to test the hypothesis.

As in normotensive diabetic rats [16], BP remained salt resistant in these hypertensive rats. The substantial renal injury displayed the histological characteristics of hypertensive injury and was linearly dependent on the pressure burden. Although classic diabetic nephropathy was not observed, the presence of diabetes mellitus increased the susceptibility of the non-clipped kidney to hypertensive renal disease. However, the susceptibility was not reduced by increased salt intake. Autoregulation was efficient in all groups. Consequently we have falsified the starting hypotheses that increased salt intake would reduce susceptibility to hypertensive injury in diabetes and that it would act via increased efficiency of autoregulation.

Salt sensitivity and BP Salt sensitivity of BP in hypertensive models is variable. Typically endothelin-dependent models are salt sensitive [31], whereas angiotensin II (ANG II)dependent models such as $2 \mathrm{~K} 1 \mathrm{C}[31,32]$ and the infarction model of 5/6 nephrectomy [33] are not. Available data with respect to salt sensitivity in diabetes are inconsistent, and the salt loads vary widely in both amount and duration. Thus, an intact rat eating $\sim 35 \mathrm{~g} /$ day of chow containing $1 \%$ salt by weight consumes $\sim 6 \mathrm{mmol} /$ day of salt; similarly, a diabetic rat eating $50-55 \mathrm{~g} /$ day of chow consumes $\sim 9 \mathrm{mmol} /$ day of salt. Consequently a diabetic rat receiving an $8 \%$ salt diet should consume $\sim 72 \mathrm{mmol} /$ day. Often, however, actual food and/or salt intakes are not reported, making it difficult to compare across studies.

Long-term telemetry studies, including this one, consistently show stable or reduced BP after induction of type 1 diabetes [14, 16, 34-37]. Recent results indicate that rats with STZ-induced diabetes show salt-resistant BP $[16,38]$ even on a background of hypertension induced by chronic inhibition of nitric oxide synthase (NOS) [38]. Salt-sensitive BP tends to be reported in studies that report hypertension induced by diabetes per se and in studies that use particularly large salt loads. Equally, when very large salt loads are used, even the control animals may show salt-sensitive BP [39]. In our hands, such a response is associated with aversive avoidance of salty fluid and food, which casts doubt on the relevance of the BP increase to the condition (type 1 diabetes) being studied. In any case, our purpose was to test the effect of changing salt intake on the type 1 diabetesinduced increase in susceptibility of the kidney to hypertensive damage. Salt-resistant BP thus makes the Long-Evans rat an excellent experimental model.

Renal injury - type 1 diabetes and salt The dominant injury shown by histology was focal and segmental glomerulosclerosis, which was significantly related to systolic pressure.
Glomerular injury in diabetic rats was more severe than in intact rats. Modest tubular atrophy and interstitial fibrosis reinforced the primacy of glomerular injury. The clipped kidneys showed minor injury, which did not differ among groups and did not correlate with BP or blood glucose. Thus the rats showed hypertensive injury, not classic diabetic nephropathy, consistent with previous reports $[8,40]$. An injury versus pressure plot, such as Fig. 6, may underestimate the degree to which a treatment increases susceptibility because terminal studies are performed first in animals with more rapidly progressive injury. Terminal studies in intact rats were performed on average a week later in the protocol than those in diabetic rats, although the protocol duration did not differ ( $\chi^{2}$ test). Diabetic rats exhibited faster progression of proteinuria, hypertension-induced renal disease at lower pressures than intact rats, and greater glomerular injury scores at the same pressure load. We interpret these findings to show a substantial increase in susceptibility to hypertensive renal disease induced by diabetes. Furthermore, there was no effect of increased salt intake, either beneficial or deleterious, in the injury-pressure plot (Fig. 6).

Intrarenal ANG II has been implicated in the development of nephropathy, particularly in salt-sensitive models [41, 42]. Renal renin and angiotensinogen contents increase in diabetes [43]. Although plasma renin activity is similar in Zucker diabetic fatty and Zucker lean rats [13], preliminary data from the same group show elevated intrarenal ANG II content in Zucker diabetic fatty rats, which is appropriately reduced by high salt intake [44]. In 2K1C clipped kidney, the renin content shows an early massive rise, but later decreases to control levels, while that of the non-clipped kidney becomes vanishingly small [45]. Thus, the clipped kidney is exposed to more renin, similar or higher ANG II, the same glycaemia, but lower BP than the non-clipped kidney. BP was independent of salt intake in our diabetic rats (Fig. 2), suggesting appropriate suppression of ANG II [46] in IN-H and DM-H. Glomerulosclerosis occurred only in the non-clipped kidney and varied with BP and diabetes, but not with salt intake. Thus, there is an apparent paradox, whereby a presumed reduction of ANG II by salt did not reduce damage. We cannot infer whether renal damage would have been reduced with pharmacological inhibition of the renin-angiotensin system or if that could be achieved without reducing BP. Although the present data cannot be generalised to state that ANG II is not involved in diabetic nephropathy, they suggest at most a minor pathogenic role.

Autoregulation-type 1 diabetes and salt An obvious question is whether the salt load was sufficient to affect pre-glomerular resistance. We cannot answer this directly because measurement of individual kidney GFR was not feasible during the chronic portion of the experiment and not meaningful at the terminal study when the non-clipped 
kidneys were variably damaged. However, there is indirect evidence that the salt load was sufficient to affect preglomerular resistance. In our previous study, the high salt intake had two significant effects: it reduced diabetic hyperfiltration and it abrogated the left shift of the lower limit of autoregulation seen in DM-N. The simplest explanation encompasses both observations. The left shift of the lower limit of autoregulation is mediated by intrarenal ANG II [29, 47]. Diabetic hyperfiltration involves increased fractional proximal reabsorption resulting in increased renin secretion. The salt-induced reduction in fractional reabsorption increases salt delivery to the macula densa [48] and reduces renin secretion. We attribute salt-induced abrogation of the left shift to reduced intrarenal ANG II. A corollary is that, in diabetic rats, the high salt intake will have caused some degree of afferent vasoconstriction mediated by TG feedback. In the present study, we see the same left shift of the lower limit of autoregulation in DM-N, and again not in $\mathrm{DM}-\mathrm{H}$. Therefore we presume that the high salt diet reduced intrarenal ANG II concentration and increased pre-glomerular resistance via TG feedback.

The results are not consistent with the hypothesis that increasing salt intake reduces susceptibility by restoring the efficiency of autoregulation. Instead, efficient autoregulation was seen in all groups. In fact, RBF was entirely independent of BP within the autoregulatory range. From this we infer that glomerular capillary pressure was effectively stabilised when BP increased and fluctuated. Thus type 1 diabetes did not impair autoregulation, which therefore cannot account for the type 1 diabetes-induced increase in susceptibility to hypertensive renal damage. This raises the question of how type 1 diabetes increases susceptibility to hypertensive renal injury when the only known protective mechanism (autoregulation) is functioning so well.

One possibility is that glomerular capillary pressure is regulated around a higher value in type 1 diabetes. Data in the literature are consistent with such a regulated increase in glomerular capillary pressure. Very different dependency of RBF dynamics on nitric oxide generation is seen in early and established diabetes. One week after STZ, RBF dynamics were essentially pressure-passive, but active myogenic and TG feedback dynamics were restored by NOS inhibition, consistent with a dominant role for nitric oxide at this time [49]. In contrast, strong myogenic and TG feedback dynamics were seen 4 weeks after STZ and were not modulated by NOS inhibition [16]. Thus, reduced renal neuronal NOS activity in established type 1 diabetes may remove dilator influence from the efferent arteriole [50] and could account for an increase in regulated glomerular capillary pressure.

In summary, this study has identified and characterised the increased susceptibility to hypertensive renal disease that is due to type 1 diabetes. It shows that type 1 diabetes does not increase susceptibility by exacerbating hypertension or by reducing efficiency of autoregulation. It shows that increased salt intake sufficient to correct diabetic hyperfiltration does not reduce susceptibility. Within the resolution of the study, no deleterious consequences of increased salt intake were found. We conclude that, despite the salt paradox, increased salt intake does not reduce renal susceptibility to hypertensive renal disease because autoregulation was never impaired. We suggest that, whether or not diabetes increases the incidence of salt sensitivity of BP, it certainly increases the susceptibility to target organ damage in hypertension and thus exacerbates the consequences of high salt intake in those diabetic patients who happen to be salt sensitive.

Acknowledgements The authors thank A.K. Bidani (Hines VA Hospital, Maywood IL, USA) for informative and entertaining discussions.

Funding The study was funded by a Grant in Aid from the Heart \& Stroke Foundation of British Columbia and Yukon and by a grant from the Victoria Foundation to W.A. Cupples. B. Braam is a Heart \& Stroke Foundation of Canada New Investigator. C.A. Sima was supported by a University of Victoria Graduate Open Scholarship, and M.P. Koeners was supported by a Kolff fellowship from the Dutch Kidney Foundation and an International travel grant from the Dutch Diabetes Foundation.

Duality of interest The authors declare that there is no duality of interest associated with this manuscript.

Contribution statement WAC conceived the study, and was the primary supervisor of the work and drafting of the manuscript. CAS and MPK performed the experiments, including data analysis, and CAS drafted the manuscript. JAJ suggested and performed several analyses. $\mathrm{BB}$ contributed to design, analysis, interpretation. ABM assessed and interpreted renal pathology. All authors contributed to editing of the manuscript and all approved the version to be published.

\section{References}

1. United States Renal Data System, USRDS (2009) Annual data report: atlas of chronic kidney disease and end-stage renal disease in the United States. National Institutes of Health, National Institute of Diabetes and Digestive and Kidney Diseases, Bethesda, MD

2. Hostetter TH (1992) Diabetic nephropathy. Metabolic versus hemodynamic considerations. Diabetes Care 15:1205-1215

3. Chen GM, McAlister FA, Walker RL, Hemmelgarn BR, Campbell NRC (2011) Cardiovascular outcomes in Framingham participants with diabetes: the importance of blood pressure. Hypertension $57: 891-865$

4. Glassock RJ, Cohen AH, Adler S (1994) Primary glomerular diseases. In: Brenner BM (ed) Brenner and Rector's the kidney. Saunders, Philadelphia

5. Perneger TV, Klag MJ, Whelton PK, Coresh J (1998) Familial aggregation of renal disease in a population-based case-control study. J Am Soc Nephrol 9:1270-1276

6. Freedman BI, Bowden DW, Rich SS, Appel RG (1998) Genetic initiation of hypertensive and diabetic nephropathy. Am J Hypertens 11:251-257 
7. Griffin KA, Abu-Naser M, Abu-Amarah I, Picken M, Williamson GA, Bidani AK (2007) Dynamic blood pressure load and nephropathy in the ZSF1 (fa/fa(cp)) model of type 2 diabetes. Am J Physiol-Ren Physiol 293:F1605-F1613

8. Zatz R, Meyer TW, Rennke HG, Brenner BM (1985) Predominance of hemodynamic rather than metabolic factors in the pathogenesis of diabetic glomerulopathy. Proc Natl Acad Sci USA 82:5963-5967

9. Cupples WA, Braam B (2007) Assessment of renal autoregulation. Am J Physiol-Ren Physiol 292:F1105-F1123

10. Bidani AK, Griffin KA (2004) Pathophysiology of hypertensive renal damage: implications for therapy. Hypertension 44:595-601

11. Loutzenhiser R, Griffin KA, Bidani AK (2006) Systolic blood pressure as the trigger for the renal myogenic response: protective or autoregulatory? Curr Opin Nephrol Hypertens 15:41-49

12. Vallon V, Blantz RC, Thomson S (2003) Glomerular hyperfiltration and the salt paradox in early type 1 diabetes mellitus: a tubulocentric view. J Am Soc Nephrol 14:530-537

13. Takenaka $\mathrm{T}$, Inoue $\mathrm{T}$, Okada $\mathrm{H}$ et al (2011) Altered gap junctional communication and renal haemodynamics in Zucker fatty rat model of type 2 diabetes. Diabetologia 54:2192-2201

14. Bidani AK, Picken M, Hacioglu R, Williamson G, Griffin KA (2007) Spontaneously reduced blood pressure load in the rat streptozotocin-induced diabetes model: potential pathogenetic relevance. Am J Physiol-Ren Physiol 292:F647-F654

15. Mauer SM, Brown DM, Steffes MW, Azar S (1990) Studies of renal autoregulation in pancreatectomized and streptozotocin diabetic rats. Kidney Int 37:909-917

16. Lau C, Sudbury I, Thomson M, Howard PL, Magil AB, Cupples WA (2009) Salt-resistant blood pressure and salt-sensitive renal autoregulation in chronic streptozotocin diabetes. Am J Physiol Regul Integr Comp Physiol 296:R1761-R1770

17. Christensen KL, Parving HH (2004) Autoregulation of glomerular filtration rate in patients with diabetes. In: Mogensen CE (ed) The kidney and hypertension in diabetes mellitus. Taylor and Francis, London, pp 495-514

18. Vallon V, Wead LM, Blantz RC (1995) Renal hemodynamics and plasma and kidney angiotensin II in established diabetes mellitus in rats: effect of sodium and salt restriction. J Am Soc Nephrol 5:1761-1767

19. Miller JA (1997) Renal responses to sodium restriction in patients with early diabetes mellitus. J Am Soc Nephrol 8:749-755

20. Luik PT, Hoogenberg K, van Der Kleij FG et al (2002) Short-term moderate sodium restriction induces relative hyperfiltration in normotensive normoalbuminuric type I diabetes mellitus. Diabetologia 45:535-541

21. Vallon V, Blantz R, Thomson S (2005) The salt paradox and its possible implications in managing hypertensive diabetic patients. Curr Hypertens Rep 7:141-147

22. Sullivan JM (1991) Salt sensitivity: definition, conception, methodology, and long-term issues. Hypertension 17:I61-I68

23. Feldt-Rasmussen B, Mathiesen ER, Deckert T et al (1987) Central role for sodium in the pathogenesis of blood pressure changes independent of angiotensin, aldosterone and catecholamines in type-1 (insulin dependent) diabetes mellitus. Diabetologia 30:610-617

24. Gerdts E, Svarstad E, Myking OL, Lund-Johansen P, Omvik P (1996) Salt sensitivity in hypertensive type-1 diabetes mellitus. Blood Pressure 5:78-85

25. Griffin KA, Bidani AK (2004) Hypertensive renal damage: insights from animal models and clinical relevance. Curr Hypertens Rep 6:145-153

26. Yagi K, Kim S, Wanibuchi H, Yamashita T, Yamamura Y, Iwao H (1997) Characteristics of diabetes, blood pressure, and cardiac and renal complications in Otsuka Long-Evans Tokushima Fatty rats. Hypertension 29:728-735
27. Llamas B, Lau C, Cupples WA, Rainville ML, Souzeau E, Deschepper CF (2006) Genetic determinants of systolic and pulse pressure in an intercross between normotensive inbred rats. Hypertension 48:921-926

28. Attia DM, Verhagen AM, Stroes ES et al (2001) Vitamin E alleviates renal injury, but not hypertension, during chronic nitric oxide synthase inhibition in rats. J Am Soc Nephrol 12:2585-2593

29. Cupples WA (1993) Angiotensin II conditions the slow component of autoregulation of renal blood flow. Am J Physiol 264:F515-F522

30. Simson EL, Gold RM (1982) The Lee obesity index vindicated? Physiol Behav 29:371-376

31. Schiffrin EL (2000) Endothelin: role in experimental hypertension. J Cardiovasc Pharmacol 35:S33-S35

32. Jackson CA, Navar LG (1986) Arterial pressure and renal function in 2-kidney, one clip Goldblatt hypertensive rats maintained on a high salt intake. J Hypertens 4:215-221

33. Daniels BS, Hostetter TH (1990) Adverse effects of growth in the glomerular microcirculation. Am J Physiol 258:F1409F1416

34. Hicks KK, Seifen E, Stimers JR, Kennedy RH (1998) Effects of streptozotocin-induced diabetes on heart rate, blood pressure and cardiac autonomic nervous control. J Auton Nerv Syst 69:21-30

35. Tatchum-Talom R, Gopalakrishnan V, McNeill JR (2000) Radiotelemetric monitoring of blood pressure and mesenteric arterial bed responsiveness in rats with streptozotocin-induced diabetes. Can J Physiol Pharmacol 78:721-728

36. Sasser JM, Sullivan JC, Hobbs JL et al (2007) Endothelin A receptor blockade reduces diabetic renal injury via an antiinflammatory mechanism. J Am Soc Nephrol 18:143-154

37. Gross V, Tank J, Partke HJ et al (2008) Cardiovascular autonomic regulation in non-obese diabetic (NOD) mice. Auton Neurosci 138:108-113

38. Brands MW, Bell TD, Fleming C, Labazi H, Sturgis LC (2007) Lack of blood pressure salt-sensitivity supports a preglomerular site of action of nitric oxide in type I diabetic rats. Clin Exp Pharmacol Physiol 34:475-479

39. Orie NN, Aloamaka CP, Iyawe VI (1994) Enhanced sensitivity to salt-induced high blood pressure in diabetes mellitus. Res Exp Med (Berl) 194:197-202

40. Janssen U, Riley SG, Vassiliadou A, Floege J, Phillips AO (2003) Hypertension superimposed on type II diabetes in Goto-Kakizaki rats induces progressive nephropathy. Kidney Int 63:2162-2170

41. Kobori H, Nangaku M, Navar LG, Nishiyama A (2007) The intrarenal renin-angiotensin system: from physiology to the pathobiology of hypertension and kidney disease. Pharmacol Rev 59:251-287

42. Vallon V (2011) Pathophysiology of the diabetic kidney. In: Comprehensive physiology. Wiley: New York. pp 1175-1232

43. Anderson S, Jung FF, Ingelfinger JR (1993) Renal reninangiotensin system in diabetes - functional, immunohistochemical, and molecular biological correlations. Am J Physiol 265: F477-F486

44. Takenaka T, Inoue T, Miyazaki T et al (2011) Further elucidation of mechanisms mediating reduced autoregulatory tone in diabetes. J Am Soc Nephrol 22:350A, Abstract

45. El-Dahr SS, Dipp S, Guan S, Navar LG (1993) Renin, angiotensinogen, and kallikrein gene expression in two-kidney Goldblatt hypertensive rats. Am J Hypertens 6:914-919

46. De'Oliveira JM, Price DA, Fisher ND et al (1997) Autonomy of the renin system in type II diabetes mellitus: dietary sodium and renal hemodynamic responses to ACE inhibition. Kidney Int $52: 771-777$ 
47. Sorensen CM, Leyssac PP, Skøtt O, Holstein-Rathlou NH (2000) Role of the renin-angiotensin system in regulation and autoregulation of renal blood flow. Am J Physiol Regul Integr Comp Physiol 279:R1017-R1024

48. Vallon V, Richter K, Blantz RC, Thomson S, Osswald H (1999) Glomerular hyperfiltration in experimental diabetes mellitus: potential role of tubular reabsorption. J Am Soc Nephrol 10:2569-2576
49. Bell TD, DiBona GF, Biemiller R, Brands MW (2008) Continuously measured renal blood flow does not increase in diabetes if nitric oxide synthesis is blocked. Am J Physiol-Ren Physiol 295: F1449-F1456

50. Bachmann S, Bosse HM, Mundel P (1995) Topography of nitric oxide synthesis by localizing constitutive NO synthases in mammalian kidney. Am J Physiol 268:F885-F898 